\title{
Stability of sets of stochastic functional differential equations with impulse effect
}

\section{Yan Xu and Zhimin He*}

${ }^{*}$ Correspondence:

hezhimin@csu.edu.cn

School of Mathematics and

Statistics, Central South University,

Changsha, Hunan 410083, People's

Republic of China

\section{Springer}

\begin{abstract}
In this paper, we study the stability of sets for a class of impulsive stochastic functional differential equations. By employing piecewise continuous Lyapunov functions with Razumikhin methods, some sufficient conditions are established to guarantee the stability of sets of impulsive stochastic functional differential equations and we also show that the impulses play an important role in the stability of stochastic functional differential equations. Three examples are presented to illustrate the effectiveness of the results obtained.
\end{abstract}

MSC: 34K20; 34K45; 34K50

Keywords: stability of sets; Brownian motion; stochastic functional differential equations; impulse; Lyapunov function; Razumikhin methods

\section{Introduction}

During the past few decades, the stability theory of stochastic differential equations and impulsive differential equations has been developed very quickly; see for instance [1-15]. A lot of stability criteria on impulsive stochastic differential equations have also been reported (see [16-23] and the references therein). Almost all of them mainly focus on the stability of the zero solution, but there is very little of research addressing the stability of sets.

The concept of stability of sets of nonlinear systems, which includes as a special case stability in the sense of Lyapunov (see Krasovskii [24]; Rouche et al. [25]), such as stability of the trivial solution, stability of the solution, stability with respect to part of the variables and so on, has become one of the most important issues in the stability theory of nonlinear systems [26-28]. The theoretical works of the stability of sets with respect to nonlinear ordinary differential equations may be traced back to Yoshizawa [29-31] in the previous century. The research to the stability of sets of impulsive differential equations can be found in [15, 32-35]. For stochastic differential equations and impulsive stochastic differential equations, we refer the reader to $[11,36-39]$ and the references therein.

In this paper, we shall extend the Razumikhin method developed in $[7,14,40]$ to investigate the stability of sets for a class of impulsive stochastic functional differential equations. Meanwhile, our results show that the impulsive effects play an important part in the stability for stochastic functional differential equations, that is, an unstable stochastic delay system can be successfully stabilized by impulses.

(c) $2015 \mathrm{Xu}$ and $\mathrm{He}$. This article is distributed under the terms of the Creative Commons Attribution 4.0 International License (http://creativecommons.org/licenses/by/4.0/), which permits unrestricted use, distribution, and reproduction in any medium, provided you give appropriate credit to the original author(s) and the source, provide a link to the Creative Commons license, and indicate if changes were made. 
The rest of this paper is organized as follows. Some preliminary notes are given in Section 2. Several theorems on stability of sets of impulsive stochastic functional differential equation are established in Section 3. In Section 4, three examples are presented to illustrate the applications of the results obtained.

\section{Preliminaries}

Throughout this paper, we use the following notations.

Let $\left(\Omega, \mathcal{F},\left\{\mathcal{F}_{t}\right\}_{t \geq 0}, P\right)$ be a complete probability space with a natural filtration $\left\{\mathcal{F}_{t}\right\}_{t \geq 0}$ satisfying the usual conditions (i.e. it is right continuous and $\mathcal{F}_{0}$ contains all $P$-null sets), and $E[\cdot]$ stand for the correspondent expectation operator with respect to the given probability measure $P$. Let $W(t)=\left(W_{1}(t), \ldots, W_{m}(t)\right)^{T}$ be an $m$-dimensional Wiener process defined on a complete probability space with a natural filtration. Let $|\cdot|$ denote the Euclidean norm in $\mathbb{R}^{n}$.

Let $\tau>0$ and $P C\left([-\tau, 0] ; \mathbb{R}^{n}\right)=\left\{\phi:[-\tau, 0] \rightarrow \mathbb{R}^{n} \mid \phi(t)\right.$ is continuous everywhere except at the points $t=t_{k} \in\left[t_{0}, \infty\right), \phi\left(t_{k}^{+}\right)$and $\phi\left(t_{k}^{-}\right)$exist with $\left.\phi\left(t_{k}^{+}\right)=\phi\left(t_{k}\right)\right\}$ with the norm $\|\phi\|=\sup _{-\tau \leq \theta \leq 0}|\phi(\theta)|$, where $\phi\left(t^{+}\right)$and $\phi\left(t^{-}\right)$denote the right-hand and left-hand limits of function $\phi(t)$ at $t$.

Denote $P C_{\mathcal{F}_{0}}^{b}\left([-\tau, 0] ; \mathbb{R}^{n}\right)$ by the family of all bounded, $\mathcal{F}_{0}$-measurable, $P C\left([-\tau, 0] ; \mathbb{R}^{n}\right)$ valued random variables. For $p>0$, denote by $P C_{\mathcal{F}_{t}}^{p}\left([-\tau, 0] ; \mathbb{R}^{n}\right)$ the family of all $\mathcal{F}_{t}$-measurable $P C\left([-\tau, 0] ; \mathbb{R}^{n}\right)$-valued random variables $\phi$ such that $E\|\phi\|^{p}<\infty$.

In this paper, we shall consider the following impulsive stochastic functional differential equation:

$$
\left\{\begin{array}{l}
d x(t)=f\left(t, x_{t}\right) d t+g\left(t, x_{t}\right) d W(t), \quad t \geq t_{0}, t \neq t_{k} \\
\Delta x\left(t_{k}\right)=I_{k}\left(t_{k}, x\left(t_{k}^{-}\right)\right), \quad t=t_{k}, k \in \mathbb{Z}^{+} \\
x_{t_{0}}(s)=\xi(s), \quad s \in[-\tau, 0]
\end{array}\right.
$$

where $\mathbb{Z}^{+}$is the set of all positive integers, $\xi=\{\xi(s):-\tau \leq s \leq 0\} \in P C_{\mathcal{F}_{0}}^{b}\left([-\tau, 0] ; \mathbb{R}^{n}\right), x(t)=$ $\left[x_{1}(t), x_{2}(t), \ldots, x_{n}(t)\right]^{T}$, and $x_{t}=\{x(t+\theta):-\tau \leq \theta \leq 0\}, x\left(t_{k}^{-}\right)=\lim _{h \rightarrow 0^{-}} x\left(t_{k}+h\right), x\left(t_{k}\right)=$ $\lim _{h \rightarrow 0^{+}} x\left(t_{k}+h\right), t_{k}(k=1,2, \ldots)$ are impulsive moments satisfying $0 \leq t_{0}<t_{1}<\cdots<t_{k}<$ $t_{k+1}<\cdots$ with $\lim _{k \rightarrow+\infty} t_{k}=+\infty, \Delta x\left(t_{k}\right)=x\left(t_{k}^{+}\right)-x\left(t_{k}^{-}\right)=x\left(t_{k}\right)-x\left(t_{k}^{-}\right)$represents the jump in the state $x$ at $t_{k}$ with $I_{k}$ determining the size of the jump. $f:\left[t_{0}, \infty\right) \times P C\left([-\tau, 0] ; \mathbb{R}^{n}\right) \rightarrow \mathbb{R}^{n}$ and $g:\left[t_{0}, \infty\right) \times P C\left([-\tau, 0] ; \mathbb{R}^{n}\right) \rightarrow \mathbb{R}^{n \times m}$ are Borel measurable, and $I_{k} \in C\left(\mathbb{R}^{+} \times \mathbb{R}^{n}, \mathbb{R}^{n}\right)$.

Definition 2.1 An $\mathbb{R}^{n}$-valued stochastic process $x(t)$ is called a solution of the problem (2.1) corresponding to initial value $\sigma$, if

(i) $x$ : $[\sigma-\tau, \sigma+\beta)$ for some $\beta(0<\beta \leq \infty)$ is continuous for $t \in[\sigma-\tau, \sigma+\beta) \backslash\left\{t_{k}: k=1,2, \ldots\right\}, x\left(t_{k}^{+}\right)$and $x\left(t_{k}^{-}\right)$exist with $x\left(t_{k}^{+}\right)=x\left(t_{k}\right)$ for $t_{k} \in[\sigma-\tau, \sigma+\beta)$, and $\left\{x_{t}\right\}_{t \geq t_{0}}$ is $\mathcal{F}_{t}$-adapted;

(ii) $\left\{f\left(t, x_{t}\right)\right\} \in L^{1}\left(\left[t_{0}, \infty\right] ; \mathbb{R}^{n}\right)$ and $\left\{g\left(t, x_{t}\right)\right\} \in L^{2}\left(\left[t_{0}, \infty\right] ; \mathbb{R}^{n \times m}\right)$;

(iii) $x(t)$ satisfies $(2.1)$.

We denote the solution of the initial problem (2.1) by $x(t ; \sigma, \xi)$, and we denote by [ $\sigma-$ $\tau, \sigma+\beta)$ the maximal right interval in which the solution $x(t ; \sigma, \xi)$ is defined.

Let $M \subset\left[t_{0}-\tau, \infty\right) \times \mathbb{R}^{n}$. We introduce the following notations:

$$
\begin{aligned}
& M(t)=\left\{x \in \mathbb{R}^{n}:(t, x) \in M\right\}, \quad t \in\left[t_{0}-\tau, \infty\right) \\
& M(t, \epsilon)=\left\{x \in \mathbb{R}^{n}: d(x, M(t))<\epsilon, \epsilon>0\right\},
\end{aligned}
$$


where

$$
d(x, M(t))=\inf _{y \in M(t)} E|x-y|
$$

is the distance between $x$ and the set $M(t)$;

$$
M_{0}(t, \epsilon)=\left\{\varphi \in P C\left([-\tau, 0] ; \mathbb{R}^{n}\right): d_{0}(\varphi, M(t))<\epsilon, \epsilon>0\right\},
$$

where

$$
d_{0}(\varphi, M(t))=\max _{s \in[-\tau, 0]} d(\varphi(s), M(t+s)) \quad \text { and } \quad \varphi \in P C\left([-\tau, 0] ; \mathbb{R}^{n}\right)
$$

We assume that the following conditions $\left(\mathrm{H}_{1}\right)-\left(\mathrm{H}_{4}\right)$ are satisfied, so that the initial value problem (2.1) has one unique solution.

$\left(\mathrm{H}_{1}\right)$ For all $\psi \in P C\left([-\tau, 0] ; \mathbb{R}^{n}\right)$ and $k \in \mathbb{Z}^{+}$, the limits

$$
\lim _{(t, \varphi) \rightarrow\left(t_{k}^{-}, \psi\right)} f(t, \varphi)=f\left(t_{k}^{-}, \psi\right), \quad \lim _{(t, \varphi) \rightarrow\left(t_{k}^{-}, \psi\right)} g(t, \varphi)=g\left(t_{k}^{-}, \psi\right)
$$

exist.

$\left(\mathrm{H}_{2}\right) f$ and $g$ satisfy the locally Lipschitz condition in $\phi$ on each compact set in $P C([-\tau, 0]$; $\left.\mathbb{R}^{n}\right)$. More precisely, for every $a \in\left[t_{0}, \sigma+\beta\right)$ and every compact set $G \in P C([-\tau, 0]$; $\left.\mathbb{R}^{n}\right)$, there exists a constant $L=L(a, G)$ such that

$$
|f(t, \varphi)-f(t, \psi)| \vee|g(t, \varphi)-g(t, \psi)| \leq L\|\varphi-\psi\|,
$$

whenever $t \in\left[t_{0}, a\right)$ and $\varphi, \psi \in G$.

$\left(\mathrm{H}_{3}\right)$ For any $\rho>0$ there exists $0<\rho_{1} \leq \rho$, such that

$$
x \in M\left(t, \rho_{1}\right) \quad \text { implies that } \quad x+I_{k}\left(t_{k}, x\right) \in M(t, \rho)
$$

for all $k \in \mathbb{Z}^{+}$.

$\left(\mathrm{H}_{4}\right) f\left(t, x_{t}\right), g\left(t, x_{t}\right) \in P C\left(\left[t_{0}, \infty\right), \mathbb{R}^{n}\right)$ for $x_{t} \in P C\left([\sigma-\tau, \infty), \mathbb{R}^{n}\right)$.

For any $t \geq t_{0}$ and $\kappa \geq 0$, let $P C_{\kappa}=\left\{\phi \in P C\left([-\tau, 0] ; \mathbb{R}^{n}\right):\|\phi\| \leq \kappa\right\}$.

We shall say that condition (A) is fulfilled if the following conditions hold:

$\left(\mathrm{A}_{1}\right)$ for each $t \in\left[t_{0}, \infty\right)$ the set $M(t)$ is not empty;

$\left(\mathrm{A}_{2}\right)$ for any compact subset $F$ of $\left[t_{0}, \infty\right) \times \mathbb{R}^{n}$ there exists a constant $K>0$ depending on $F$ such that if $(t, x),\left(t^{\prime}, x\right) \in F$, then the following inequality holds:

$$
\left|d(x, M(t))-d\left(x, M\left(t^{\prime}\right)\right)\right| \leq K\left|t-t^{\prime}\right|
$$

$\left(\mathrm{A}_{3}\right)$ if for solution $x(t ; \sigma, \xi)$ there exists $h>0$ satisfying

$$
d(x(t ; \sigma, \xi), M(t, \rho)) \leq h<\infty \quad \text { for } t \in[\sigma, \sigma+\beta),
$$

where $\rho$ is a constant, then $x(t ; \sigma, \xi)$ is defined in the interval $[\sigma, \infty)$. 
Definition 2.2 A function $V(t, x):\left[t_{0}-\tau, \infty\right) \times M(t, \rho) \rightarrow \mathbb{R}^{+}$belongs to the class $v_{0}$ if

$\left(\mathrm{B}_{1}\right) V$ is continuous on each of the set $\left(\left[t_{0}-\tau, t_{0}\right] \cup\left[t_{k-1}, t_{k}\right)\right) \times M(t, \rho)$ for all $x \in M(t, \rho)$

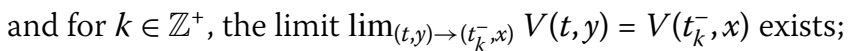

$\left(\mathrm{B}_{2}\right) V$ is locally Lipschitz in $x \in M(t, \rho), V(t, 0)=0$ for $(t, x) \in M$ and $V(t, x)>0$ for $(t, x) \notin M$.

Definition 2.3 For each $V \in v_{0}$, we define the operator $L V$ from $\mathbb{R}^{+} \times \mathbb{R}^{n}$ to $\mathbb{R}$ by

$$
\begin{aligned}
L V(t, \phi)= & V_{t}(t, x)+V_{x}(t, x) f(t, \phi) \\
& +\frac{1}{2} \operatorname{trace}\left[g^{T}(t, \phi) V_{x x}(t, x) g(t, \phi)\right],
\end{aligned}
$$

where

$$
\begin{aligned}
& V_{t}(t, x)=\frac{\partial V(t, x)}{\partial t}, \\
& V_{x}(t, x)=\left(\frac{\partial V(t, x)}{\partial x_{1}}, \ldots, \frac{\partial V(t, x)}{\partial x_{n}}\right), \\
& V_{x x}(t, x)=\left(\frac{\partial^{2} V(t, x)}{\partial x_{i} \partial x_{j}}\right)_{n \times n} .
\end{aligned}
$$

We shall give the definitions of stability of the set $M$ with respect to system (2.1).

Definition 2.4 The set $M$ with respect to the solution of system (2.1) is said to be:

$\left(\mathrm{S}_{1}\right)$ stable, if for any $\sigma \geq t_{0}, \alpha>0$, and $\epsilon>0$, there is a $\delta(\sigma, \epsilon, \alpha)>0$ such that $\xi \in P C_{\alpha} \cap$ $M_{0}(\sigma, \delta)$ implies that $x(t, \sigma, \xi) \in M(t, \epsilon)$ for $t \geq \sigma$;

$\left(\mathrm{S}_{2}\right)$ uniformly stable, if the $\delta$ in $\left(\mathrm{S}_{1}\right)$ is independent of $\sigma$;

$\left(\mathrm{S}_{3}\right)$ asymptotically stable, if it is stable and for any $\sigma \geq t_{0}$ and $\alpha>0$, there exists a $\delta=$ $\delta(\sigma, \alpha)$ such that $\xi \in P C_{\alpha} \cap M_{0}(\sigma, \delta)$ implies that $x(t, \sigma, \xi) \rightarrow M(t)$ as $t \rightarrow \infty$;

$\left(\mathrm{S}_{4}\right)$ uniformly asymptotically stable, if it is uniformly stable, and for any $\alpha>0$ there exists a $\delta(\alpha)>0$, such that for any $\epsilon>0$ there is a $T(\epsilon, \alpha, \delta)>0$ such that $\sigma \geq t_{0}$ and $\xi \epsilon$ $P C_{\alpha} \cap M_{0}(\sigma, \delta)$ implies that $x(t, \sigma, \xi) \in M(t, \epsilon)$ for $t \geq \sigma+T$.

In order to obtain our results, we will use the following function classes:

$$
\begin{aligned}
& K_{1}=\left\{u \in C\left(\mathbb{R}^{+}, \mathbb{R}^{+}\right): u(0)=0, u(s) \text { is strictly increasing in } s\right\} \\
& K_{2}=\left\{u \in C\left(\mathbb{R}^{+}, \mathbb{R}^{+}\right): u(0)=0, u(s)>0 \text { for } s>0\right\} \\
& K_{3}=\left\{u \in C\left(\mathbb{R}^{+}, \mathbb{R}^{+}\right): u(0)=0, u(s)>s \text { for } s>0, u(s) \text { is strictly increasing in } s\right\} .
\end{aligned}
$$

\section{Main results}

In this section, we present and prove our main results on uniform stability and asymptotic stability of the sets of system (2.1) by utilizing piecewise continuous Lyapunov functions with Razumickhin methods.

Theorem 3.1 Let conditions (A) and $\left(\mathrm{H}_{1}\right)-\left(\mathrm{H}_{4}\right)$ be satisfied and suppose that there exist functions $V \in v_{0}, a, b \in K_{1}, c \in K_{2}, P \in K_{3}$, and the following conditions are fulfilled: 
(i) $a(d(x, M(t))) \leq E V(t, x) \leq b(d(x, M(t)))$ for all $(t, x) \in\left[t_{0}-\tau, \infty\right) \times M(t, \rho)$;

(ii) $E L V(t, x(t)) \leq \eta(t) c(E V(t, x(t))), t \neq t_{k}$, whenever $E V(t+s, x(t+s)) \leq P(E V(t, x(t)))$ for $-\tau \leq s \leq 0$, where $x(t)$ is any solution of system $(2.1)$, and $\eta:\left[t_{0}, \infty\right) \rightarrow \mathbb{R}^{+}$is locally integrable;

(iii) $E V\left(t_{k}, x+I_{k}\left(t_{k}, x\right)\right) \leq P^{-1}\left(E V\left(t_{k}^{-}, x\right)\right)$ for each $k \in \mathbb{Z}^{+}$, and all $x \in M\left(t, \rho_{1}\right)$, where $P^{-1}$ is the inverse of the function $P$;

(iv) $\sup _{k \in \mathbb{Z}^{+}}\left\{t_{k}-t_{k-1}\right\}<\infty$, and $\int_{P^{-1}(\mu)}^{\mu} \frac{d s}{c(s)}-\int_{t_{k-1}}^{t_{k}} \eta(s) d s>0$ for all $\mu \in(0, \infty), k \in \mathbb{Z}^{+}$.

Then the set $M$ is uniformly stable with respect to the solution of system (2.1).

Proof For any given $\epsilon>0, \alpha>0$, without loss of generality, we assume that $\epsilon \leq \rho_{1}$. We can choose $\delta=\delta(\epsilon, \alpha)>0$ such that $P(b(\delta))<\alpha(\epsilon)$ and $\delta<\alpha$. From $b(\delta)<P(b(\delta))<\alpha(\epsilon)<b(\epsilon)$ we know that $\delta<\epsilon$.

For $\sigma \geq t_{0}, \xi \in P C_{\alpha} \cap M_{0}(\sigma, \delta)$, let $x(t)=x(t ; \sigma, \xi)$ be the solution of system (2.1), where $\sigma \in\left[t_{m-1}, t_{m}\right)$ for some $m \in \mathbb{Z}^{+}$. Then, for $\sigma-\tau \leq t \leq \sigma$, from condition (i) we have

$$
a(d(x(t), M(t))) \leq E V(t, x(t)) \leq b(d(x(t), M(t))) \leq b(\delta) \leq P(b(\delta))<a(\epsilon) .
$$

From the above inequality, we obtain $d(x(t), M(t))<\epsilon$ for $\sigma-\tau \leq t \leq \sigma$.

Next, we will prove $d(x(t), M(t))<\epsilon$ for $t \in[\sigma, \sigma+\beta)$. Suppose, on the contrary, that $d(x(t), M(t))>\epsilon$ for some $t \in[\sigma, \sigma+\beta)$. Then let $\hat{t}=\inf \{\sigma \leq t \leq \sigma+\beta \mid d(x(t), M(t))>\epsilon\}$. Note that $d(x(\sigma), M(\sigma))<\epsilon$, we see that $\hat{t}>\sigma, d(x(t), M(t)) \leq \epsilon \leq \rho_{1}$, for $t \in[\sigma-\tau, \hat{t})$ and either $d(x(\hat{t}), M(\hat{t}))=\epsilon$ or $d(x(\hat{t}), M(\hat{t}))>\epsilon$ and $\hat{t}=t_{k}$ for some $k$.

In the latter case, $d(x(\hat{t}), M(\hat{t})) \leq \rho$. From condition $\left(\mathrm{H}_{3}\right)$ we have

$$
d(x(\hat{t}), M(\hat{t}))=d\left(x\left(t_{k}\right), M\left(t_{k}\right)\right)=d\left(x\left(t_{k}^{-}\right)+I_{k}\left(t_{k}, x\left(t_{k}^{-}\right)\right), M\left(t_{k}\right)\right) \leq \rho,
$$

it follows that in either case $E V(t, x(t))$ is defined for $t \in[\sigma-\tau, \hat{t}]$.

For $t \in[\sigma, \hat{t}]$ define

$$
E V(t)=E V(t, x(t))
$$

Then for $t \in[\sigma-\tau, \hat{t}]$, by condition (i), we get

$$
a(d(x(t), M(t))) \leq E V(t) \leq b(d(x(t), M(t))) .
$$

Let $\tilde{t}=\inf \{t \in[\sigma, \hat{t}] \mid E V(t)>a(\epsilon)\}$. Since $E V(\sigma)<a(\epsilon)$ and $E V(\tilde{t}) \geq a(\epsilon)$, it follows that $\tilde{t} \in(\sigma, \hat{t}]$ and $E V(t)<a(\epsilon)$ for $t \in[\sigma-\tau, \tilde{t})$. We claim that $E V(\tilde{t})=a(\epsilon)$ and that $\tilde{t} \neq t_{k}$ for any $k$. In fact, if $E V(\tilde{t}) \geq a(\epsilon), \tilde{t}=t_{k}$ for some $k$, by condition (iii) we have

$$
a(\epsilon) \leq E V(\tilde{t}) \leq P^{-1}\left(E V\left(\tilde{t}^{-}\right)\right)<E V\left(\tilde{t}^{-}\right) \leq a(\epsilon)
$$

which is contradiction. Thus $\tilde{t} \neq t_{k}$, for any $k$, and that in turn implies $E V(\tilde{t})=a(\epsilon)$, since $E V(t)$ is continuous at $\tilde{t}$ for $\tilde{t} \neq t_{k}$.

Now let us first consider the case $t_{m-1} \leq \tilde{t}<t_{m}$. Let $\bar{t}=\sup \left\{t \in[\sigma, \tilde{t}] \mid E V(t) \leq P^{-1}(a(\epsilon))\right\}$. Since $E V(\sigma)<P^{-1}(a(\epsilon)), E V(\tilde{t})=a(\epsilon)>P^{-1}(a(\epsilon))$, and $E V(t)$ is continuous on $[\sigma, \tilde{t}]$, we have $\bar{t} \in(\sigma, \tilde{t}), E V(\bar{t})=P^{-1}(a(\epsilon))$, and $E V(t) \geq P^{-1}(a(\epsilon))$ for $t \in[\bar{t}, \tilde{t}]$. 
For $t \in[\bar{t}, \tilde{t}]$ and $-\tau \leq s \leq 0$, we have

$$
E V(t+s) \leq a(\epsilon)=P\left(P^{-}(a(\epsilon))\right) \leq P(E V(t)) .
$$

From condition (ii), we obtain

$$
E L V(t) \leq \eta(t) c(E V(t))
$$

for all $t \in[\bar{t}, \tilde{t}]$. Integrating the above differential inequality yields

$$
\int_{E V(\bar{t})}^{E V(\tilde{t})} \frac{d s}{c(s)} \leq \int_{\bar{t}}^{\tilde{t}} \eta(s) d s \leq \int_{t_{m-1}}^{t_{m}} \eta(s) d s
$$

On the other hand, by condition (iv), we obtain

$$
\int_{E V(\bar{t})}^{E V(\tilde{t})} \frac{d s}{c(s)}=\int_{P^{-1}(a(\epsilon))}^{a(\epsilon)} \frac{d s}{c(s)}>\int_{t_{m-1}}^{t_{m}} \eta(s) d s
$$

which is in contradiction with (3.3).

Now, assume that $t_{k}<\tilde{t}<t_{k+1}$ for some $k \in \mathbb{Z}^{+}$and $k \geq m$. Then by condition (iii) we have

$$
E V\left(t_{k}\right) \leq P^{-1}\left(E V\left(t_{k}^{-}\right)\right)<P^{-1}(a(\epsilon)) .
$$

Let $\bar{t}=\sup \left\{t \in\left[t_{k}, \tilde{t}\right] \mid E V(t) \leq P^{-1}(a(\epsilon))\right\}$. Then $\bar{t} \in\left(t_{k}, \tilde{t}\right), E V(\bar{t})=P^{-1}(a(\epsilon))$, and $E V(t) \geq$ $P^{-1}(a(\epsilon))$ for $t \in[\bar{t}, \tilde{t}]$. Therefore, for $t \in[\bar{t}, \tilde{t}]$ and $-\tau \leq s \leq 0$, we have

$$
E V(t+s) \leq a(\epsilon)=P\left(P^{-}(a(\epsilon))\right) \leq P(E V(t)) .
$$

Then, by condition (ii), we have

$$
E L V(t) \leq \eta(t) c(E V(t)) \quad \text { for all } t \in[\bar{t}, \tilde{t}] .
$$

Integrating the above differential inequality yields

$$
\int_{E V(\bar{t})}^{E V(\tilde{t})} \frac{d s}{c(s)} \leq \int_{\bar{t}}^{\tilde{t}} \eta(s) d s \leq \int_{t_{k}}^{t_{k+1}} \eta(s) d s .
$$

On the other hand, by condition (iv), we have

$$
\int_{E V(\bar{t})}^{E V(\tilde{t})} \frac{d s}{c(s)}=\int_{P^{-1}(a(\epsilon))}^{a(\epsilon)} \frac{d s}{c(s)}>\int_{t_{k}}^{t_{k+1}} \eta(s) d s,
$$

which is in contradiction with (3.4). So in either case, we get a contradiction, so we obtain

$$
d(x(t, \sigma, \xi), M(t))<\epsilon \quad \text { for } t \in[\sigma, \sigma+\beta) .
$$


From condition $\left(\mathrm{A}_{3}\right)$ we know that $[\sigma, \sigma+\beta)=[\sigma, \infty)$, hence $x(t) \in M(t, \epsilon)$, for all $t \geq \sigma$, which implies that the set $M$ is uniformly stable with respect to the solution of system (2.1). The proof of Theorem 3.1 is complete.

Remark 3.1 From Theorem 3.1, we know that impulsive perturbations may cause uniform stability even if the unperturbed system is unstable.

The following result on the asymptotical stability of sets will reveal that impulsive perturbation make stable systems asymptotically stable.

Theorem 3.2 Let conditions (A) and $\left(\mathrm{H}_{1}\right)-\left(\mathrm{H}_{4}\right)$ be satisfied and suppose that there exist functions $V \in v_{0}, a, b \in K_{1}, h_{k} \in C\left(\mathbb{R}^{+}, \mathbb{R}^{+}\right)$for $k \in \mathbb{Z}^{+}$, and the following conditions are fulfilled:

(i) $a(d(x, M(t))) \leq E V(t, x) \leq b(d(x, M(t)))$ for all $(t, x) \in\left[t_{0}-\tau, \infty\right) \times M(t, \rho)$;

(ii) $E V\left(t_{k}, x+I_{k}\left(t_{k}, x\right)\right)-E V\left(t_{k}^{-}, x\right) \leq-h_{k}\left(E V\left(t_{k}^{-}, x\right)\right)$ for all $k \in \mathbb{Z}^{+}$and $x \in M\left(t, \rho_{1}\right)$;

(iii) for any solution $x(t)$ of system (2.1), $E L V(t, x) \leq 0$;, and for any $\sigma \geq t_{0}$, and $r>0$, there exists $\left\{r_{k}\right\}$ such that $E V(t, x) \geq r$ for $t \geq \sigma$ implies that $h_{k}\left(E V\left(t_{k}^{-}, x\right)\right) \geq r_{k}$; where $r_{k} \geq 0$ with $\sum_{k=1}^{\infty} r_{k}=\infty$.

Then the set $M$ with respect to the solution of system (2.1) is uniformly stable and asymptotically stable.

Proof At first, we show that the set $M$ is uniform stability.

For given $\epsilon>0\left(\epsilon \leq \rho_{1}\right), \alpha>0$, we choose a $\delta(\epsilon, \alpha)>0$ such that $b(\delta) \leq a(\epsilon)$ and $\delta<\alpha$. For any $\sigma \geq t_{0}$ and $\xi \in P C_{\alpha} \cap M_{0}(\sigma, \delta)$, let $x(t)=x(t ; \sigma, \xi)$ be the solution of system (2.1). We will show that $x(t) \in M(t, \epsilon)$ for $t \in[\sigma, \sigma+\beta)$.

Set $E V(t)=E V(t, x(t))$, where $\sigma \in\left[t_{m-1}, t_{m}\right)$ for some $m \in \mathbb{Z}^{+}$. Then condition (iii) implies that $E L V(t) \leq 0$ for $t \in[\sigma, \sigma+\beta) \cap\left(\left[\sigma, t_{m}\right) \cup\left(\bigcup_{k=m}^{\infty}\left[t_{k-1}, t_{k}\right)\right)\right), k \in \mathbb{Z}^{+}$.

By condition (ii) we have $E V\left(t_{i}\right)-E V\left(t_{i}^{-}\right) \leq 0$ for all $\sigma \leq t_{i} \leq \sigma+\beta$. Thus $E V(t)$ is nonincreasing on $[\sigma, \sigma+\beta)$. From condition (i) it follows that

$$
a(d(x(t), M(t))) \leq E V(t) \leq E V(\sigma) \leq b(\delta) \leq a(\epsilon)
$$

for $\sigma \leq t \leq \sigma+\beta$. From condition $\left(\mathrm{A}_{3}\right)$ we obtain $[\sigma, \sigma+\beta)=[\sigma, \infty)$. Since $d(x(t), M(t)) \leq$ $\epsilon$, for all $t \geq \sigma$, this implies that $x(t) \in M(t, \epsilon)$ for $t \geq \sigma$. That is, the set $M$ is uniformly stable with respect to the solution of system (2.1).

Next we shall prove that the set $M$ is asymptotically stable.

From conditions (ii), (iii), and $E V(t) \geq 0$, we note that $E V(t)$ is non-increasing on the interval $[\sigma, \infty)$. So the limit $\lim _{t \rightarrow \infty} E V(t)$ exists.

Assume $\sigma \in\left[t_{m-1}, t_{m}\right]$ for some $m \in \mathbb{Z}^{+}$. Set $\lim _{t \rightarrow \infty} E V(t)=r \geq 0$, one can easily see that $E V(t) \geq r$ for $t \geq \sigma$. Then by condition (iii), it follows that there is a sequence $\left\{r_{k}\right\}$ with $r_{k} \geq 0$ for $k \in \mathbb{Z}^{+}$, which implies that $h_{k}\left(E V\left(t_{k}^{-}, x\right)\right) \geq r_{k}$ with $\sum_{k=1}^{\infty} r_{k}=\infty$.

By conditions (ii) and (iii) we get

$$
\begin{aligned}
E V(t) & \leq E V(\sigma)+\sum_{\sigma \leq t_{k} \leq t}\left(E V\left(t_{k}\right)-E V\left(t_{k}^{-}\right)\right) \\
& \leq E V(\sigma)-\sum_{\sigma \leq t_{k} \leq t} h_{k}\left(E V\left(t_{k}^{-}\right)\right) \\
& \leq E V(\sigma)-\sum_{\sigma \leq t_{k} \leq t} r_{k} \rightarrow-\infty \quad(t \rightarrow \infty),
\end{aligned}
$$


which is a contradiction. Hence we have $r=0$, which implies that $a(d(x, M(t))) \rightarrow 0$ as $t \rightarrow \infty$. That is, $x(t) \rightarrow M(t)$ as $t \rightarrow \infty$. The proof of Theorem 3.2 is complete.

Theorem 3.3 Let conditions (A) and $\left(\mathrm{H}_{1}\right)-\left(\mathrm{H}_{4}\right)$ be satisfied and suppose that there exist functions $V \in v_{0}, a, b \in K_{1}, \psi_{k}, C \in K_{2}$, and the following conditions are fulfilled:

(i) $a(d(x, M(t))) \leq E V(t, x) \leq b(d(x, M(t)))$ for all $(t, x) \in\left[t_{0}-\tau, \infty\right) \times M(t, \rho)$;

(ii) $E V\left(t_{k}, x+I_{k}\left(t_{k}, x\right)\right) \leq \psi_{k}\left(E V\left(t_{k}^{-}, x\right)\right)$, for all $K \in \mathbb{Z}^{+}$, and $x \in M(t, \rho)$;

(iii) for any solution $x(t)$ of system (2.1), $E L V(t, x) \leq-\theta(t) C(E V(t, x))$ for $t \neq t_{k}$, where $\theta:\left[t_{0}, \infty\right) \rightarrow \mathbb{R}^{+}$is locally intergrade, and there exists $\mu_{0}$, such that for any $\mu \in\left(0, \mu_{0}\right)$,

$$
\int_{\mu}^{\psi_{k}(\mu)} \frac{d s}{C(s)}-\int_{t_{k-1}}^{t_{k}} \theta(s) d s \leq-\gamma_{k}
$$

where $\gamma_{k} \geq 0$ with $\sum_{k=1}^{\infty} \gamma_{k}=\infty$.

Then the set $M$ with respect to the solution of system (2.1) is uniformly stable and asymptotically stable.

Proof Without loss of generality, for any given $\epsilon>0, \alpha>0$, we can assume that $\epsilon \leq \rho_{1}$. We choose a $\beta: 0<\beta<\min \left\{a(\epsilon), \mu_{0}\right\}$ such that $\psi_{k}(s)<a(\epsilon)$ for $0 \leq s \leq \beta$ and for all $k \in \mathbb{Z}^{+}$.

Set $\delta=\delta(\epsilon, \alpha)>0$ be such that $b(\delta)<\beta$ and $\delta<\alpha$. Let $x(t)=x(t ; \sigma, \xi)$ be the solution of system (2.1), where $\sigma \geq t_{0}$ and $\xi \in P C_{\alpha} \cap M_{0}(\sigma, \delta)$. At first, we show that

$$
x(t) \in M(t, \epsilon) \text { for } t \in[\sigma, \sigma+\beta) .
$$

Set $E V(t)=E V(t, x(t))$ and $\sigma \in\left[t_{m-1}, t_{m}\right)$ for some $m \in \mathbb{Z}^{+}$.

By condition (iii), we get $E L V(t, x) \leq 0$ for $\sigma \leq t<t_{m}$. It follows that

$$
E V(t) \leq E V(\sigma) \leq b(\delta)<\beta<a(\epsilon)
$$

for $\sigma \leq t<t_{m}$. So for $\sigma \leq t<t_{m}$, we have $x(t) \in M(t, \epsilon)$. Thus if (3.5) is not true, then there exists a $\bar{t} \in\left[t_{k}, t_{k+1}\right)$ for some $k \in \mathbb{Z}^{+}, k \geq m$ such that $x(t) \in M(t, \epsilon)$ for $\sigma \leq t<\bar{t}$, and $x(\bar{t}) \notin M(\bar{t}, \epsilon)$. Using conditions (ii) and (iii), we have, for $i=m, m+1, \ldots, k-1$,

$$
E L V(t) \leq-\theta(t) C(E V(t)), \quad t_{i} \leq t<t_{i+1}
$$

and

$$
E V\left(t_{i}\right) \leq \psi_{i}\left(E V\left(t_{i}^{-}\right)\right)
$$

So by (3.7), we have

$$
E V\left(t_{m}\right) \leq \psi_{m}\left(E V\left(t_{m}^{-}\right)\right) \leq \psi_{m}(b(\delta))<a(\epsilon) .
$$

From (3.6) and (3.7), for $i=m, m+1, \ldots, k-1$, we have

$$
\int_{E V\left(t_{i}\right)}^{E V\left(t_{i+1}^{-}\right)} \frac{d s}{C(s)} \leq-\int_{t_{i}}^{t_{i+1}} \theta(s) d s
$$


and

$$
\int_{E V\left(t_{i+1}^{-}\right)}^{E V\left(t_{i+1}\right)} \frac{d s}{C(s)} \leq \int_{E V\left(t_{i+1}^{-}\right)}^{\psi\left(E V\left(t_{i+1}^{-}\right)\right)} \frac{d s}{C(s)} .
$$

Thus by (3.9) and condition (iii), we obtain

$$
\int_{E V\left(t_{i}\right)}^{E V\left(t_{i+1}\right)} \frac{d s}{C(s)} \leq \int_{E V\left(t_{i+1}^{-}\right)}^{\psi\left(E V\left(t_{i+1}^{-}\right)\right)} \frac{d s}{C(s)}-\int_{t_{i}}^{t_{i+1}} \theta(s) d s \leq-\gamma_{i+1},
$$

which implies $E V\left(t_{i+1}\right) \leq E V\left(t_{i}\right)$ for $i=m, m+1, \ldots, k-1$. From this and (3.8) we have

$$
E V\left(t_{k}\right) \leq \cdots \leq E V(m)<a(\epsilon) .
$$

But by condition (i), we have $a(\epsilon) \leq a(d(x(\bar{t}), M(\bar{t}))) \leq E V(\bar{t}) \leq E V\left(t_{k}\right)<a(\epsilon)$, which is a contradiction. Thus (3.5) holds, from condition $\left(\mathrm{A}_{3}\right)$ it follows that $(\sigma-\tau, \sigma+\beta)=(\sigma-$ $\tau, \infty)$, hence $x(t) \in M(t, \epsilon)$, for all $t \geq \epsilon$. So the set $M$ is uniformly stable with respect to the solution of system (2.1).

To prove the asymptotically stability, we observe that, from the proof of (3.11), one finds that $E V\left(t_{i+1}\right) \leq E V\left(t_{i}\right)$ holds for all $i \geq m$. Thus we have $\lim _{t \rightarrow \infty} E V\left(t_{i}\right)=\alpha$ exists and $\alpha \geq 0$. If $\alpha>0$, (3.11) yields

$$
\int_{E V\left(t_{i}\right)}^{E V\left(t_{i+1}\right)} \frac{d s}{C(s)} \leq-\gamma_{i+1}, \quad i=m, m+1, \ldots
$$

Let $\bar{c}=\inf _{\alpha \leq s<a(\epsilon)} C(s)$. From (3.13), we get

$$
E V\left(t_{i+1}\right) \leq E V\left(t_{i}\right)-\bar{c} \gamma_{i+1}, \quad i=m, m+1, \ldots,
$$

which implies

$$
E V\left(t_{k}\right) \leq E V\left(t_{m}\right)-\bar{c} \sum_{i=m}^{k-1} \gamma_{i+1} \rightarrow-\infty
$$

as $k \rightarrow \infty$. It is a contradiction and so $\alpha=0$.

Since $E V(t) \leq E V\left(t_{k}\right)$ for $t_{k} \leq t<t_{k+1}$, it follows that $\lim _{t \rightarrow \infty} E V(t)=0$, which yields $\lim _{t \rightarrow \infty} d(x(t, M(t)))=0$. The proof of Theorem 3.3 is complete.

\section{Illustrative examples}

As an application, we consider the following examples.

Example 4.1 Consider the scalar impulsive stochastic delay differential equation:

$$
\left\{\begin{array}{l}
d x(t)=(-x(t)+1.2 x(t-\tau)) d t+\frac{1}{\sqrt{10}} x(t-\tau) d W(t), \quad t \neq t_{k}, \\
x\left(t_{k}\right)=0.5 x\left(t_{k}^{-}\right), \quad k=1,2, \ldots,
\end{array}\right.
$$

where $\tau>0, t_{0}<t_{1}<t_{2}<\cdots<t_{k} \rightarrow \infty$ as $k \rightarrow \infty$. Assume that the following condition is satisfied:

$$
t_{k}-t_{k-1}<-\frac{\ln 0.5}{1.6}, \text { for } k \in \mathbb{Z}^{+} \text {, where } t_{0} \geq 0 .
$$


Let $M(t)=\left\{(t, 0): t \in\left[t_{0}-\tau, \infty\right)\right\}, V(t, x)=V(x)=0.5 x^{2}, P(s)=4 s, c(s)=s$, then

$$
E V\left(x+I_{k}\left(t_{k}, x\right)\right)=E V(0.5 x)=E\left(0.125 x^{2}\right)=P^{-1}(E V(x)),
$$

and for any solution $x(t)$ of system (4.1), such that

$$
E V(t+s, x(t+s)) \leq P(E V(x(t))), \quad-\tau \leq s \leq 0, t \geq t_{0} .
$$

Clearly, we have $E x^{2}(t-\tau) \leq 4 E x^{2}(t), t \geq t_{0}$. Hence,

$$
\begin{aligned}
E L V(x(t)) & =-E x^{2}(t)+1.2 E x(t) x(t-\tau)+0.5 \times 0.1 E x^{2}(t-\tau) \\
& \leq-E x^{2}(t)+2.4 E x^{2}(t)+0.2 E x^{2}(t) \\
& =\eta(t) c(E V(x(t)))
\end{aligned}
$$

where $\eta(t)=3.2>0$.

We have

$$
t_{k}-t_{k-1}<-\frac{\ln 0.5}{1.6}
$$

and for any $\mu>0, k \in \mathbb{Z}^{+}$,

$$
\begin{aligned}
\int_{P^{-1}(\mu)}^{\mu} \frac{d s}{c(s)}-\int_{t_{k-1}}^{t_{k}} \eta(s) d s & =\int_{h^{2} \mu}^{\mu} \frac{d s}{c(s)}-\int_{t_{k-1}}^{t_{k}} \eta(s) d s \\
& >-2 \ln 0.5-\left(-\frac{\ln 0.5}{1.6}\right) \times 2 \times(1.6) \\
& =0 .
\end{aligned}
$$

Thus all of the conditions in Theorem 3.1 are satisfied. Therefore, it follows from Theorem 3.1 that the set $M$ is uniformly stable with respect to the solution of the system (4.1). The simulation result of system (4.1) is shown in Figure 1. The simulation of system (4.1) without impulses is shown in Figure 2. From Figures 1 and 2, we find that, although stochastic delay differential equations without impulse may be unstable, adding impulses may lead to stability. That is, impulsive perturbations play an important role in the stability behavior of nonlinear systems.

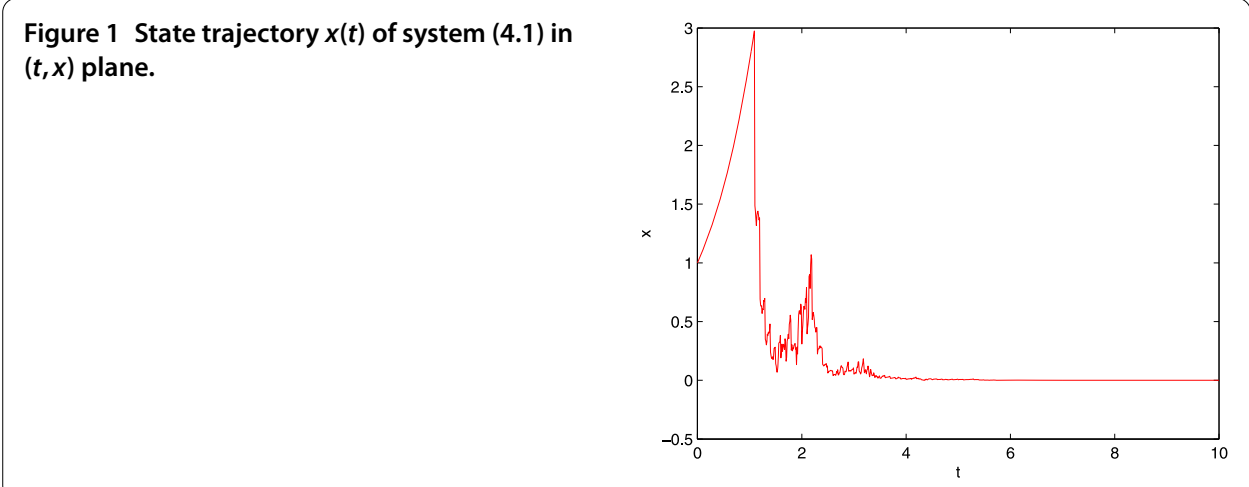




\section{Figure 2 State trajectory $x(t)$ of system (4.1)} without impulses in $(t, x)$ plane.

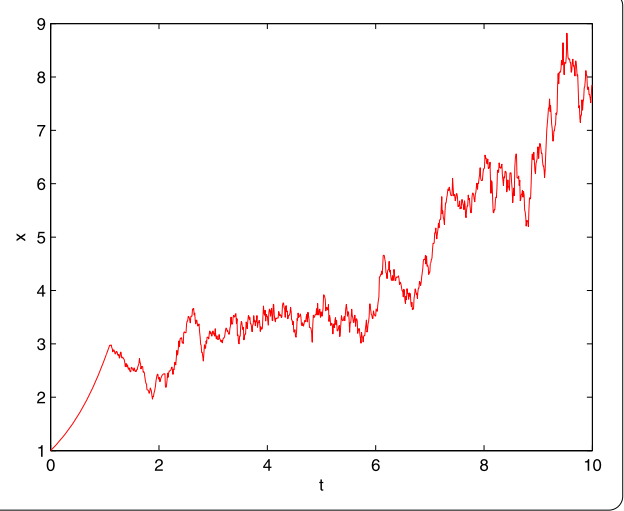

Example 4.2 Consider the scalar impulsive stochastic delay differential equation:

$$
\left\{\begin{array}{l}
d x(t)=(m x(t)+n x(t-\tau)) d t+(p x(t)+q x(t-\tau)) d W(t), \quad t \neq t_{k} \\
x\left(t_{k}\right)=u x\left(t_{k}^{-}\right), \quad k=1,2, \ldots
\end{array}\right.
$$

where $\tau>0, t_{0}<t_{1}<t_{2}<\cdots<t_{k} \rightarrow \infty$ as $k \rightarrow \infty$. Assume that the following condition is satisfied:

$$
0<u<1 \text { and } m+|n| u^{-1}+\frac{1}{2} p^{2}+|p q| u^{-1}+\frac{1}{2} q^{2} u^{-2}<0 .
$$

Let $M(t)=\left\{(t, 0): t \in\left[t_{0}-\tau, \infty\right)\right\}, V(t, x)=V(x)=\frac{1}{2} x^{2}, h_{k}(s)=\left(1-u^{2}\right) s$, then

$$
\begin{aligned}
& E V\left(x+I_{k}\left(t_{k}, x\right)\right)=E V(u x)=E\left(\frac{1}{2} u^{2} x^{2}\right), \\
& E V\left(t_{k}, x+I_{k}\left(t_{k}, x\right)\right)-E V\left(t_{k}^{-}, x\right)=-\left(1-u^{2}\right) \frac{1}{2} x^{2}=-h_{k}\left(E V\left(t_{k}^{-}, x\right)\right) .
\end{aligned}
$$

Clearly, we have $E x^{2}(t-\tau) \leq h^{-2} E x^{2}(t), t \geq t_{0}$. Hence,

$$
\begin{aligned}
E L V(x(t)) & =m E x^{2}(t)+n E x(t) x(t-\tau)+\frac{1}{2} p^{2} E x^{2}(t)+p q E x(t) x(t-\tau)+\frac{1}{2} q^{2} E x^{2}(t-\tau) \\
& \leq m E x^{2}(t)+|n| u^{-1} E x^{2}(t)+\frac{1}{2} p^{2} E x^{2}(t)+|p q| u^{-1} E x^{2}(t)+\frac{1}{2} q^{2} u^{-2} E x^{2}(t) \\
& =2\left(m+|n| u^{-1}+\frac{1}{2} p^{2}+|p q| u^{-1}+\frac{1}{2} q^{2} u^{-2}\right) E V(x(t))<0
\end{aligned}
$$

We check that for any $\sigma \geq t_{0}$, and $r>0$, there exists $\left\{r_{k}\right\}$ such that $E V(t, x) \geq r$ for $t \geq \sigma$ implies that $h_{k}\left(E V\left(t_{k}^{-}, x\right)\right) \geq r_{k}$; where $r_{k} \geq 0$ with $\sum_{k=1}^{\infty} r_{k}=\infty$. Since $h_{k}\left(E V\left(t_{k}^{-}, x\right)\right)=(1-$ $\left.u^{2}\right) E V\left(t_{k}^{-}, x\right)$, when $E V(t, x) \geq r$ for $t \geq \sigma$, then we have $h_{k}\left(E V\left(t_{k}^{-}, x\right)\right)=\left(1-u^{2}\right) E V\left(t_{k}^{-}, x\right) \geq$ $\left(1-u^{2}\right) r$. We take $r_{k}=\left(1-u^{2}\right) r$ and we have $\sum_{k=1}^{\infty} r_{k}=\infty$. Thus all of the conditions in Theorem 3.2 are satisfied. Therefore, it follows from Theorem 3.2 that the set $M$ is uniformly stable and asymptotically stable with respect to the solution of the system (4.2).

Example 4.3 Consider the scalar impulsive stochastic delay differential equation:

$$
\left\{\begin{array}{l}
d x(t)=(a x(t)+b x(t-\tau)) d t+(c x(t)+r x(t-\tau)) d W(t), \quad t \neq t_{k}, \\
x\left(t_{k}\right)=h x\left(t_{k}^{-}\right), \quad k=1,2, \ldots,
\end{array}\right.
$$


where $\tau>0, t_{0}<t_{1}<t_{2}<\cdots<t_{k} \rightarrow \infty$ as $k \rightarrow \infty$. Assume that the following conditions are satisfied:

(i) $0<h<1$ and $a+|b| h^{-1}+\frac{1}{2} c^{2}+|c r| h^{-1}+\frac{1}{2} r^{2} h^{-2}<0$;

(ii) $t_{k}-t_{k-1}>\frac{\ln h}{a+|b| h^{-1}+\frac{1}{2} c^{2}+|c| h^{-1}+\frac{1}{2} r^{2} h^{-2}}$, for $k \in \mathbb{Z}^{+}$, where $t_{0} \geq 0$.

Let $M(t)=\left\{(t, 0): t \in\left[t_{0}-\tau, \infty\right)\right\}, V(t, x)=V(x)=\frac{1}{2} x^{2}, \psi_{k}(s)=h^{2} s, C(s)=s$, then

$$
E V\left(x+I_{k}\left(t_{k}, x\right)\right)=E V(h x)=E\left(\frac{1}{2} h^{2} x^{2}\right)=\psi_{k}(E V(x))
$$

and for any solution $x(t)$ of system (4.3), such that

$$
E V(t+s, x(t+s)) \leq \psi_{k}(E V(x(t))), \quad-\tau \leq s \leq 0, t \geq t_{0} .
$$

Clearly, we have $E x^{2}(t-\tau) \leq h^{-2} E x^{2}(t), t \geq t_{0}$. Hence,

$$
\begin{aligned}
E L V(x(t)) & =a E x^{2}(t)+b E x(t) x(t-\tau)+\frac{1}{2} c^{2} E x^{2}(t)+c r E x(t) x(t-\tau)+\frac{1}{2} r^{2} E x^{2}(t-\tau) \\
& \leq a E x^{2}(t)+|b| h^{-1} E x^{2}(t)+\frac{1}{2} c^{2} E x^{2}(t)+|c r| h^{-1} E x^{2}(t)+\frac{1}{2} r^{2} h^{-2} E x^{2}(t) \\
& =-\theta(t) C(E V(x(t)))<0,
\end{aligned}
$$

where $\theta(t)=-2\left(a+|b| h^{-1}+\frac{1}{2} c^{2}+|c r| h^{-1}+\frac{1}{2} r^{2} h^{-2}\right)>0$.

We have

$$
t_{k}-t_{k-1}>\frac{\ln h}{a+|b| h^{-1}+\frac{1}{2} c^{2}+|c r| h^{-1}+\frac{1}{2} r^{2} h^{-2}}
$$

and for any $\mu>0, k \in \mathbb{Z}^{+}$,

$$
\begin{aligned}
\int_{\mu}^{\psi_{k}(\mu)} \frac{d s}{C(s)}-\int_{t_{k-1}}^{t_{k}} \theta(s) d s= & \int_{\mu}^{h^{2} \mu} \frac{d s}{C(s)}-\int_{t_{k-1}}^{t_{k}} \theta(s) d s \\
< & 2 \ln h-\frac{\ln h}{a+|b| h^{-1}+\frac{1}{2} c^{2}+|c r| h^{-1}+\frac{1}{2} r^{2} h^{-2}} \\
& \times(-2)\left(a+|b| h^{-1}+\frac{1}{2} c^{2}+|c r| h^{-1}+\frac{1}{2} r^{2} h^{-2}\right) \\
= & 4 \ln h .
\end{aligned}
$$

Letting $\gamma_{k}=-4 \ln h$, then $\gamma_{k} \geq 0$ with $\sum_{k=1}^{\infty} \gamma_{k}=\infty$. Thus all of the conditions in Theorem 3.3 are satisfied. Therefore, it follows from Theorem 3.3 that the set $M$ is uniformly stable and asymptotically stable with respect to the solution of the system (4.3).

\section{Competing interests}

The authors declare that they have no competing interests.

\section{Authors' contributions}

All authors contributed equally to the writing of this paper. All authors read and approved the final manuscript.

\section{Acknowledgements}

The authors would like to thank the referee for his/her careful reading and valuable suggestions, which lead to improvement of the manuscript.

Received: 5 December 2014 Accepted: 22 June 2015 Published online: 04 July 2015 


\section{References}

1. Bao, JH, Hou, ZT, Yuan, CG: Stability in distribution of mild solutions to stochastic partial differential equations. Proc. Am. Math. Soc. 138, 2169-2180 (2010)

2. Bao, JH, Truman, A, Yuan, CG: Stability in distribution of mild solutions to stochastic partial differential delay equations with jumps. Proc. R. Soc. Lond. Ser. A 465, 2111-2134 (2009)

3. Bao, JH, Hou, ZT, Yuan, CG: Stability in distribution of neutral stochastic differential delay equations with Markovian switching. Stat. Probab. Lett. 79, 1663-1673 (2009)

4. Khasminskii, R: Stochastic Stability of Differential Equations, 2nd edn. Springer, Berlin (2012)

5. Ladde, GS, Lakshmikantham, V: Random Differential Inequalities. Academic Press, New York (1980)

6. Ladde, GS, Sambandham, M: Stochastic versus Deterministic Systems of Differential Equations. Dekker, New York (2004)

7. Lakshmikantham, V, Bainov, DD, Simeonov, DS: Theory of Impulsive Differential Equation. World Scientific, Singapore (1989)

8. Lakshmikantham, V, Liu, X: Stability of impulsive differential systems in terms of two measures. Appl. Math. Comput. 29, 89-98 (1989)

9. Liu, X, Wang, Q: On stability in terms of two measures for impulsive systems of functional differential equations. J. Math. Anal. Appl. 326, 252-265 (2007)

10. Mao, X: Stochastic Differential Equations and Their Applications, 2nd edn. Horwood, Chichester (2007)

11. Mao, X: Attraction, stability and boundedness for stochastic differential delay equations. Nonlinear Anal. 47, 4795-4806 (2001)

12. Samoilenko, AM, Perestyuk, NA: Impulsive Differential Equations. World Scientific, Singapore (1995)

13. Shaikhet, L: Lyapunov Functionals and Stability of Stochastic Functional Differential Equations. Springer, New York (2013)

14. Shen, J, Yan, J: Razumikhin type stability theorems for impulsive functional differential equations. Nonlinear Anal. 33 , 519-531 (1998)

15. Stamova, I: Stability Analysis of Impulsive Functional Differential Equations. de Gruyter, New York (2009)

16. Caro, EA, Rao, ANV: Stability analysis of impulsive stochastic differential systems in terms of two measures. In: Conference Proceedings COM, pp. 132-135. IEEE Press, New York (1996)

17. Pan, L, Cao, J: Exponential stability of impulsive stochastic functional differential equations. J. Math. Anal. Appl. 382, $672-685(2011)$

18. Liu, J, Liu, X, Xie, W: Impulsive stabilization of stochastic functional differential equations. Appl. Math. Lett. 24, 264-269 (2011)

19. Liu, ZM, Peng, J: p-Moment stability of stochastic nonlinear delay systems with impulsive jump and Markovian switching. Stoch. Anal. Appl. 27, 911-923 (2009)

20. Peng, S, Jia, B: Some criteria on $p$ th moment stability of impulsive stochastic functional differential equations. Stat. Probab. Lett. 80, 1085-1092 (2010)

21. Rao, ANV, Tsokos, CP: Stability behavior of impulse stochastic differential systems. Dyn. Syst. Appl. 4, $317-327$ (1995)

22. Zhang, S, Sun, J, Zhang, Y: Stability of impulsive stochastic differential equations in terms of two measures via perturbing Lyapunov functions. Appl. Math. Comput. 218, 5181-5186 (2012)

23. $\mathrm{Xu}, \mathrm{Y}, \mathrm{He}, \mathrm{ZM}$ : Stability of impulsive stochastic differential equations with Markovian switching. Appl. Math. Lett. 35 35-40 (2014)

24. Krasovskii, NN: Stability of Motion. Stanford University Press, Stanford (1963)

25. Rouche, H, Habets, P, Laloy, M: Stability Theory by Lyapunov's Direct Method. Springer, New York (1977)

26. Lakshmikantham, V, Leela, S, Martynyuk, AA: Stability Analysis of Nonlinear Systems. Dekker, New York (1989)

27. Lakshmikantham, V, Leela, S, Martynyuk, AA: Practical Stability Analysis of Nonlinear Systems. World Scientific, Singapore (1990)

28. Lakshmikantham, V, Liu, X: Stability Analysis in Terms of Two Measures. World Scientific, Singapore (1993)

29. Yoshizawa, T: Stability of sets and perturbed system. Funkc. Ekvacioj 5, 31-69 (1962)

30. Yoshizawa, T: Some notes on stability of sets and perturbed system. Funkc. Ekvacioj 6, 1-11 (1964)

31. Yoshizawa, T: Stability Theory by Lyapunov's Second Method. The Mathematical Society of Japan, Tokyo (1966)

32. Kulev, GK, Bainov, DD: Stability of sets for impulsive systems. Int. J. Theor. Phys. 28(2), 195-207 (1989)

33. Kulev, GK, Bainov, DD: Global stability of sets for impulsive differential systems by Lyapunov's direct method. Comput. Math. Appl. 19, 17-28 (1990)

34. Xie, S: Stability of sets of functional differential equations with impulse effect. Appl. Math. Comput. 218, 592-597 (2011)

35. Xie, S, Shen, J: Stability of sets for impulsive functional differential equations via Razumikhin method. J. Math. Sci. 177, 474-486 (2011)

36. Long, S: Attracting and invariant sets of nonlinear stochastic neutral differential equations with delays. Results Math 63, 745-762 (2013)

37. Samoilenko, AM, Stanzhytskyi, O: Qualitative and Asymptotic Analysis of Differential Equations with Random Perturbations. World Scientific, Singapore (2011)

38. Luo, JW: Stability of invariant sets of Itô stochastic differential equations with Markovian switching. J. Appl. Math. Stoch. Anal. 2006, Article ID 59032 (2006)

39. $\mathrm{Xu}, \mathrm{LG}, \mathrm{Xu}, \mathrm{DY}$ : P-Attracting and $p$-invariant sets for a class of impulsive stochastic functional differential equations. Comput. Math. Appl. 57, 54-61 (2009)

40. Mao, X: Razumikhin type theorems on exponential stability of stochastic functional differential equations. Stoch. Process. Appl. 65, 233-250 (1996) 